\title{
Independent Component Analysis of Four-Phase Abdominal CT Images
}

\author{
Xuebin $\mathrm{Hu}^{1}$, Akinobu Shimizu ${ }^{1}$, Hidefumi Kobatake ${ }^{1}$, and Shigeru Nawano ${ }^{2}$ \\ ${ }^{1}$ Graduate School of Bio-Applications and Systems Engineering, \\ Tokyo University of Agriculture \& Technology \\ 2-24-16 Naka-cho, Koganei-shi, Tokyo, 184-8588 Japan \\ \{huxb, simiz, kobatake\}@cc.tuat.ac.jp \\ ${ }^{2}$ National Cancer Center Hospital East \\ 6-5-1 Kasiwanoha, Kasiwa-shi, Chiba, 277-8577 Japan \\ snawano@east.ncc.go.jp
}

\begin{abstract}
This paper presents a new analysis result of two-dimensional fourphase abdominal CT images using variational Bayesian mixture of ICA. The four-phase CT images are modeled as being produced by a mixture of data generators, where each data generator consists of a set of independent components and is responsible for generating a particular cluster. ICA results show that the CT images could be divided into a set of clinically and anatomically meaningful components. Initial analysis of the independent components shows its promising prospect in medical image processing and computer-aided diagnosis.
\end{abstract}

\section{Introduction}

Independent component analysis (ICA), as one of the most powerful tool in multivariate analysis, has attracted a great of interests in medical signal and image processing. Examples include in EEG, ECG de-noising, removing artifacts and signal analysis, coloring multi-channel MR imaging data, extracting blood vessel-related component from dynamic brain PET images, and analysis of functional magnetic resonance imaging (fMRI) data etc. In this paper, we attempt to use the recently developed variational Bayesian mixture of ICA (vbmoICA) [1], to analyze four-phase abdominal CT images for finding the latent meaningful components.

ICA mixture model was first formulated by Lee et al. in [2], in which it is defined as that the observed data are categorized into several mutually exclusive classes, and the data in each class are modeled as being generated by a linear combination of independent sources. It relaxes the assumption of standard ICA that the sources must be independent, and shows improved performance in data classification problems [3]. Lee et al. use the extended Infomax algorithm [4] to switch the source model between sub-Gaussian and super-Gaussian regimes, and learn parameters of the model using gradient ascent to maximize a log-likelihood function. The variational Bayesian 
mixture of ICA used in this paper differs from the Lee's method mainly in two aspects. The first is that, instead of a predefined density model, vbmoICA uses a fully adaptable mixture of Gaussians as the ICA source model, allowing complex and potentially multi-model distributions to be modeled. The second difference is that the mixtures of ICA model are trained using variational Bayesian method, which is carried through to the mixture model. This allows model comparison, incorporation of prior knowledge, control of model complexity thus avoiding over-fitting.

Four-phase abdominal CT images are taken at different phases before and after the contrast material injected. It is often used as an effective measure for tumor detection. The four-phase CT images are modeled as being produced by a mixture of data generators, where each data generator consists of a set of independent components and is responsible for generating a particular cluster. ICA mixture analysis results show that the CT images could be divided into a set of clinically and anatomically meaningful components. Initial analysis of the independent components shows the promising prospect of ICA mixture analysis in medical image processing and computer-aided diagnosis.

In the subsequent pages of this paper, the variational Bayesian mixture of ICA is briefly introduced in section 2. Section 3 presents the meaningful ICA result. In section 4, we discuss its applications with some preliminary analysis. At the last is the conclusion.

\section{Variational Bayesian Mixture of ICA}

\subsection{ICA Mixture Model}

Assume that the data $\boldsymbol{X}=\left\{\boldsymbol{x}^{n}, n=1, \cdots, N\right\}$ are drawn independently and generated by a mixture density model. $N$ is the total number of data vectors and each data vector $\boldsymbol{x}^{n}$ is of $S$-dimension. The probability of generating a data vector $\boldsymbol{x}^{n}$ from a $C$ component mixture model given assumptions $M$ is given by [1]:

$$
p\left(x^{n} \mid M\right)=\sum_{c=1}^{C} p\left(c \mid M_{0}\right) p\left(x^{n} \mid M_{c}, c\right)
$$

A data vector $\boldsymbol{x}^{n}$ is generated by choosing one of the $C$ components stochastically under $p\left(c \mid M_{0}\right)$ and then drawing from $p\left(x^{n} \mid M_{c}, c\right) . M=\left\{M_{0}, M_{1}, \cdots, M_{C}\right\}$ is the vector of component model assumption, $M_{c}$, and assumption about the mixture process, $M_{0}$. The variable $c$ indicates which component of the mixture model is chosen to generate a given data vector $\boldsymbol{x}^{n} . p\left(c \mid M_{0}\right)$ is a vector of probabilities. $p\left(x^{n} \mid M_{c}, c\right)$ is the component densities and assumed to be non-Gaussian. The mixture density $p\left(x^{n} \mid M\right)$ is known as the evidence for model $M$ and quantifies the likelihood of the observed data under model $M[1]$.

In the $C$-component (cluster) mixture model, the observed variables, $\boldsymbol{x}$, within each cluster are assumed to be generated by linearly transforming an unobserved source vector $\boldsymbol{s}_{c}$, of dimension $L_{c}$ with added Gaussian noise $\boldsymbol{e}_{c}[1]$, 


$$
\boldsymbol{x}=\boldsymbol{A}_{c} \boldsymbol{s}_{c}+\boldsymbol{y}_{c}+\boldsymbol{e}_{c}
$$

where $\boldsymbol{y}_{c}$ is an $S$-dimensional bias vector, $\boldsymbol{e}_{c}$ is $S$-dimensional additive noise and $c$ represents the $c$-th ICA model (component). In signal processing nomenclature, $S$ is the number of sensors and $L_{c}$ is the number of latent sources. Equation (2) acts as a complete description for cluster $c$ in the data density. The bias vector, $\boldsymbol{y}_{c}$, defines the position of the cluster in the $S$-dimensional data space, $\boldsymbol{A}_{c}$ describes its orientation and $\boldsymbol{s}_{c}$ describes the underlying manifold. The noise, $\boldsymbol{e}_{c}$, is assumed to be zero-mean Gaussian and isotropic with precision $\lambda_{c} \boldsymbol{I}$. The probability of observing data vector $\boldsymbol{x}^{n}$ under component $c$ is given by [1]

$$
p\left(x^{n} \mid \theta_{c}, c\right)=\left(\frac{\lambda_{c}}{2 \pi}\right)^{\frac{S}{2}} \exp \left[-E_{c}\right]
$$

where $\theta_{c}=\left\{A_{c}, s_{c}^{n}, \lambda_{c}, y_{c}\right\}$ and

$$
E_{c}=\frac{\lambda_{c}}{2}\left(\boldsymbol{x}^{n}-\boldsymbol{A}_{c} \boldsymbol{s}_{c} \boldsymbol{n}-\boldsymbol{y}_{c}\right)^{T}\left(\boldsymbol{x}^{n}-\boldsymbol{A}_{c} \boldsymbol{s}_{c} \boldsymbol{n}-\boldsymbol{y}_{c}\right)
$$

Since the sources $\boldsymbol{s}_{c}=\left\{s_{c, 1} \cdots, s_{c, i}, \cdots s_{c, L_{c}}\right\}$ are by definition mutually independent, the distribution over $s_{c}$ for data point $n$ can be written as [1]

$$
p\left(s_{c}^{n} \mid M_{S_{c}}, c\right)=\prod_{i=1}^{L_{c}} p\left(s_{c, i}^{n} \mid M_{S_{c}, i}, c\right)
$$

where the product runs over the $L_{c}$ sources of component $c$, and $M_{S_{c}}$ is the vector of source model assumptions.

In equation (5), $p\left(s_{c}^{n} \mid M_{S_{c}}, c\right)$ is the source model for ICA component $c$. It is a factorized mixture of 1-dimensional Gaussians with $L_{c}$ factors (i.e. sources) and $m_{i}$ components per source

$$
p\left(\boldsymbol{s}_{c}^{n} \mid \varphi_{c}\right)=\prod_{i=1}^{L_{c}} \sum_{q_{i}=1}^{m_{i}} \pi_{i, q_{i}} N\left(s_{c, i}^{n} ; \mu_{i, q_{i}}, \beta_{i, q_{i}}\right)
$$

Equation (6) essentially describes the local features of cluster $c, \mu_{i, q_{i}}$ is the position of feature $q_{i}, \beta_{i, q_{i}}$ is its size, and $\pi_{i, q_{i}}$ is its prominence. The mixture proportions $\pi_{i, q_{i}}$ are the prior probabilities of choosing component $q_{i}$ of the $i$-th source of the $c$-th ICA model. $q_{i}^{n}$ is a variable indicating which component of the $i$-th source is chosen for generating $s_{c, i}^{n} . \mu_{i, q_{i}}$ and $\beta_{i, q_{i}}$ are the mean and covariance of Gaussian $q_{i}$ in source $i$. The parameters of $i$-th source are $\varphi_{c, i}=\left\{\pi_{c, i}, \mu_{c, i}, \beta_{c, i}\right\}$ where bold face indicates the vector of $m_{i}$ parameters. The complete parameter set of the source model is $\varphi_{c}=\left\{\varphi_{c, 1}, \varphi_{c, 2}, \cdots, \varphi_{c, L_{c}}\right\}[1]$. 


\subsection{Variational Bayesian Learning}

The above defined ICA mixture model is trained using variational Bayesian method. The prior distributions over the hidden variables and model parameters are given based on the defined mixture model and some additional assumptions. The details are referred to [1]. Bayesian inference in such a model is computationally intensive and
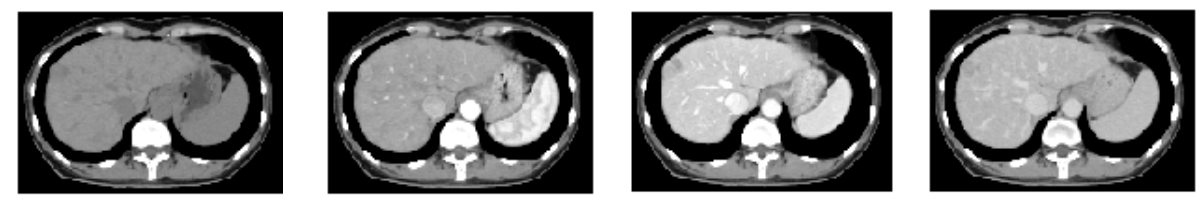

Fig. 1. Four-phase abdominal CT images. They are, from left to right, the pre-contrast image, image of early phase, portal phase, and late phase, respectively. These images are displayed within the range from -110 to $190 \mathrm{H} . \mathrm{U}$.

intractable. To overcome the problem, the variational method $[5,6]$ is used to make assumptions about the posterior distributions and give tractability to the Bayesian model. The variational Bayesian method constructs a lower bound on the marginal likelihood, and attempts to optimize this bound using an iterative scheme that has intriguing similarities to the standard expectation-maximization algorithm. The parameters of model are learnt by alternating between estimating the posterior distribution over hidden variables for a particular setting of the parameters and then re-estimating the best-fit parameters given that distribution over the hidden variables.

\section{ICA Analysis Result}

We use the variational Bayesian mixture of ICA algorithm on a set of multi-phase CT images of human abdomen. The data was provided by the National Cancer Center Hospital East, which was collected at four phases before and after contrast material injected. Figure 1 shows the four phases CT images. They are the pre-contrast, early, portal and late images, respectively. The later three phase images were firstly registered to the pre-contrast image by template matching of the center of spine [7]. Then the four 512x512 pixel images were compressed to $170 \times 170$ pixel images.

The structure of model can be decided by calculating a term called negative freeenergy (NFE) in the vbmoICA. The higher the NFE is, the better the structure that representing the data. Experimentally, we set the number of clusters equals to four, and each cluster is generated from a set of three latent independent components. The four images were vectorised, and 7000 samples were randomly drawn from the data. Then the sampled data were used to train the model. The trained models were then used to unmix the complete dataset into the corresponding independent features.

Figure 2 shows the derived independent components, which present a very meaningful result. The second class of independent components that consists of the components numbered 4-6 appear to be responsible for generating the liver, spleen and stomach areas with the muscle and fat tissue at the outside contour. The third 
class of ICs (7-9) seem to mainly highlight the ribs and spine, and the forth IC class (10-12) appear to emphasis the lower part of lung. One of the most attractive points is that, in the fifth component, the ribs and lung are completely removed, which makes the segmentation of liver or the spleen area much easier than the original CT images. Another interesting point is that there is a tumor at the left-up end of the liver, and it becomes more visible comparing to the original images.

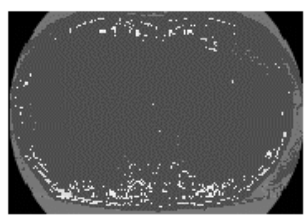

1

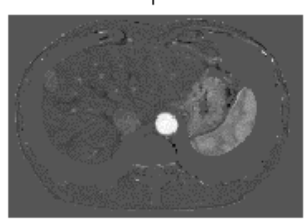

4

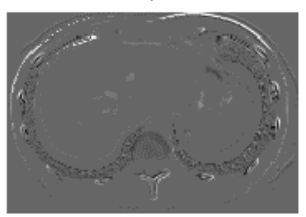

7

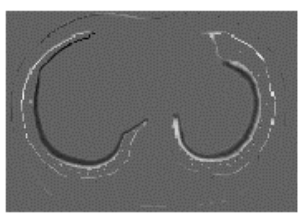

10

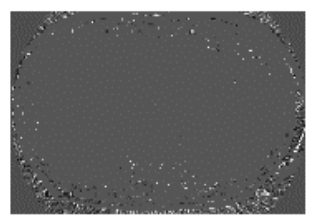

2

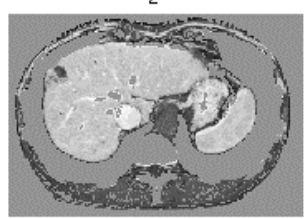

5

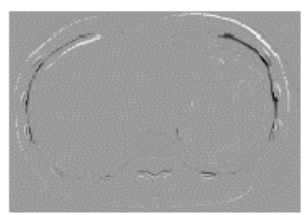

8

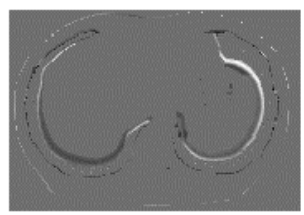

11

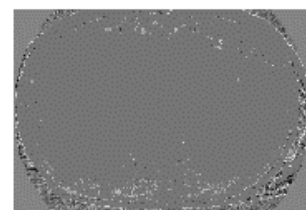

3

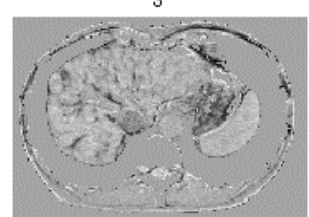

6

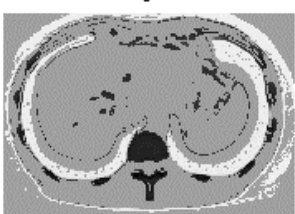

9

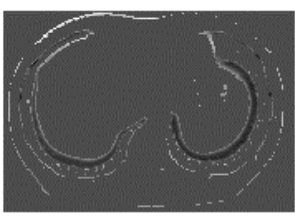

12

Fig. 2. ICA analysis result of four-phase abdominal CT images using vbmoICA. The data distribution in the vectorised CT images data is assumed to be consists of 4 clusters, and the each cluster is generated from a set of 3 latent independent components. The 4-6 ICs appear to highlight the internal organs, while the 7-9 seems to highlight the ribs and spine, and the 10-12 ICs seems to emphasis the lung. These images are displayed at their whole intensity ranges, respectively.

\section{Applications}

The anatomically and clinically meaningful result as shown in Fig.2 implies the prospects in various potential applications. Here we discuss its applications in segmentation and tumor detection. 


\subsection{Liver Area Segmentation}

Segmentation of the organs, i.e., extracting boundary elements belonging to the same structure, from medical images is more difficult compared to other imaging fields due

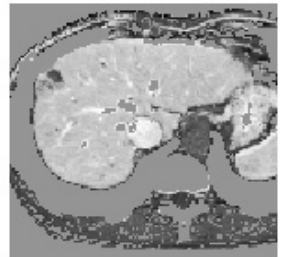

(a)

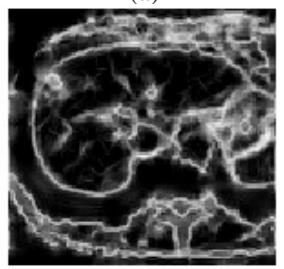

(c)

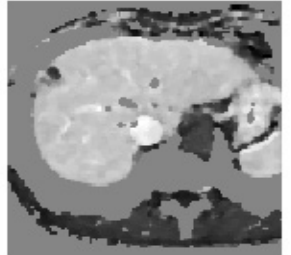

(b)

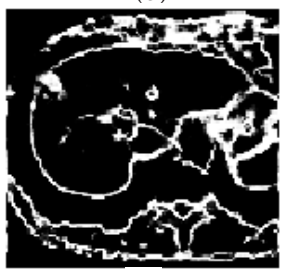

(d)

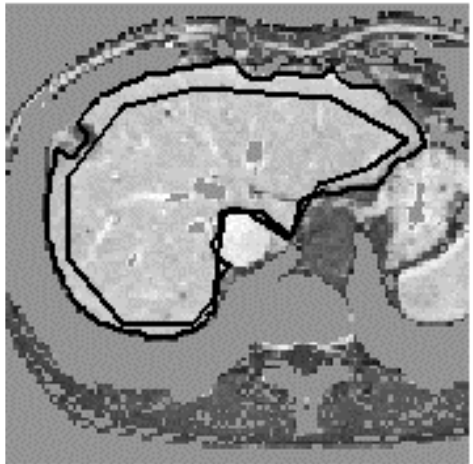

(e)

Fig. 3. Preprocessing and segmentation result. (a) is the fifth independent component, (b) is the image after smoothing, (c) is the feature image of phase congruency, (d) is the one after sigmoid filter. Figure 3(e) shows the segmentation result. The inner line shows the initial contour and the outer line shows the final reshaped contour of liver area.

to the large variability in shapes and complexity of anatomic structures. For example, the liver area is difficult to segment because surrounding tissues, such as stomach and muscle, have similar CT values and sometimes contact with each other, which may cause the boundaries of structures to be indistinct and disconnected. Although deformable models appear promising result because of their ability to match images of anatomic structures by exploiting constraints derived from the image data, it is still difficult to achieve a satisfying result. Consequently, preprocessing to simplify the complicity will be very helpful and expected.

The GVFSnake method proposed by Xu and Prince [8] is used in the segmentation test on the fifth feature. The difference of GVFSnake from the conventional Snake is that in GVFSnake the gradient vector field (GVF) is first calculated and used for increasing the capture range of the attracting image. The preprocessing includes the following three steps: 1) Smoothing by median filter (Fig.3(b)); 2) Detecting edge using phase congruency filter (Phase congruency is an illumination and contrast invariant measure of feature [9]. It is used here for emphasizing the edge reliably, see Fig.3(c)); 3) Suppressing unnecessary lines by sigmoid filter (Fig.3(d)).

After the processing, it is normal that the image does not only indicate the wanted border, but several others as well (see Fig.3(d)). There should be an initial contour that is quite close to the object to segment; otherwise it might get stuck on an irrelevant border. The initial contour may be obtained using some pixel-wised methods, for example [7]. Here we give it manually. Fig.3(e) shows the segmentation result. The inner line is the initial contour, where the outer line is the final contour. It 
shows that a good segmentation is easily achieved on the fifth component, which is often difficult on the original images. This is due to the fact that on the fifth feature, some of the tissues surrounding the liver have been removed and the contrast of CT intensities in liver area increased comparatively.

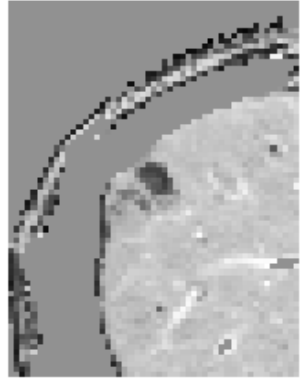

(a)

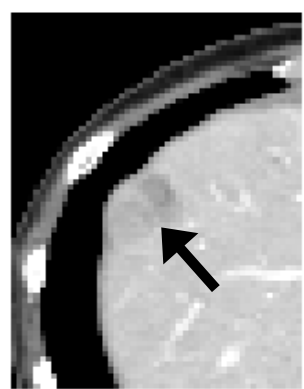

(b)

Fig. 4. Close-ups of the tumor in (a) the fifth IC, and (b) the portal phase image. The arrow in (b) indicates its location.

\subsection{Contrast Variation Around Tumor}

Another potential application of ICA analysis result is tumor detection. According to the doctor, there is a tumor at the left-up end of the liver. Fig.4(b) depicts its location. Fig.4 shows the close-ups of tumor on the fifth IC and portal phase image, respectively. It appears that the tumor has been emphasized in the fifth independent component comparing with the four original images, which means that the tumor becomes more detectable on IC than on the original images. For comparing the detectability of tumor quantitatively, we use the evaluation method [10], in which the detectability is quantified using the signal-to-noise ratio defined as below,

$$
S N R=\left(P_{\max }-\mu\right) / \sigma
$$

where, $\mu$ and $\sigma$ denote the mean and standard deviation of the intensities of pixels surrounding the tumor within $10 \mathrm{~mm}$ Euclidean distance. $P_{\max }$ denotes the maximum intensity value within the tumor area. Table 1 shows the result. The fifth IC has a higher SNR value than the original images, which means the tumor is easier to be found using the independent component.

Table 1. Tumor detectability comparison

\begin{tabular}{|c|c|c|c|c|c|}
\hline \multirow{2}{*}{} & \multicolumn{4}{|c|}{ Original CT images } & \multirow{2}{*}{$5^{\text {th }}$ IC } \\
\cline { 2 - 5 } & Pre-contrast & Early & Portal & Late & \\
\hline SNR & 3.46 & 3.20 & 7.02 & 4.46 & 13.16 \\
\hline
\end{tabular}




\section{Conclusion}

We performed the independent component analysis on several sets of data, and each achieved similar meaningful result. The reason why ICA mixture model can achieve such a meaningful results is not much clear. The explanation could be given is by Choudrey et. al. in [1] that the clusters in the model are learned to be represented by their own local coordinate systems that are constructed under the independent assumption. We once tried the standard ICA model on the CT images but failed to find any interesting phenomena. The reason is thought two: first, standard ICA has not the clustering ability; second, multi-phase CT images actually are highly similar with each other (except for the effect from the contrast material that whitens some particular organs or tissues in a sequence decided by flood circulation), i.e., in the ICA model $\boldsymbol{x}=\boldsymbol{A s}, \boldsymbol{x}$ are quite similar. This equally means $\boldsymbol{A}$ is nearly singular because each channel of the observed variables is generated under similar mixing process. In such case, the latent features are difficult to be found. On the contrary, the clustering ability of ICA mixture model decreases the difficulty because the independent features are found within each decomposed cluster.

The anatomically and clinically meaningful results make us believe the promising prospect of ICA mixture model as preprocessing to increase the performance of organ segmentation and tumor detection. Currently, we are working on expanding its application on three-dimensional abdominal CT images.

\section{References}

[1] R.A. Choudrey and S.J. Roberts, "Variational mixture of Bayesian independent component analyzers," Neural Computation, vol. 15(1), Jan. 2003.

[2] T.W. Lee, M.S. Lewicki, "Upsupervised image classification, segmentation, and enhancement using ICA mixture models," IEEE Trans. on Image Processing, vol. 11, No. 3, March 2002.

[3] T.W. Lee, M.S. Lewicki, and T.J. Sejnowski, "ICA mixture models for unsupervised classification and automatic context switching," Proceeding of International workshop on Independent Component Analysis, 1999, pp.209-214.

[4] T.W. Lee, M. Girolami, and T.J. Sejnowski, "Independent component analysis using an extended infomax algorithm for mixed sub-Gaussian and super-Gaussian sources," Neural Computation, vol. 11, no. 2, pp.409-4333, 1999.

[5] T. Jaakkola and M. Jordan, "Bayesian parameter estimation via variational methods," Statistics and Computing, vol. 10, pp. 25-37, 2000.

[6] H. Attias, "Learning parameters and structure of latent variable models by variational Bayes," in Electronic Proceedings of the Fifteenth Annual Conference on Uncertainty in Artificial Intelligence (UAI1999), http://www2.sis.pitt.edu/ rds1/UAI/ uai.html.

[7] T. Hitosugi, A. Shimizu, M. Tamura, and H. Kobatake, "Development of a liver extraction method using a level set method and its performance evaluation," Journal of Computer Aided Diagnosis of Medical Images, vol. 7, No. 4-2, Jun. 2003 (in Japanese).

[8] C. Xu and J. L. Prince, "Snakes, shapes, and gradient vector flow," IEEE Trans. on Image Processing, 7(3), pp. 359-369, Mar. 1998. 
[9] Peter Kovesi, "Image Features From Phase Congruency". Videre: A Journal of Computer Vision Research. MIT Press. Volume 1, Number 3, 1999.

[10] Y. Okada, A. Shimizu, H. Kobatake, J. Hasegawa, and J. Toriwaki, "Evaluation of SN ratio of lung tumors in maximum intensity projection images and mean intensity projection images," Medical Imaging Technology. Vol. 21, No. 2, pp. 139-146, Mar. 2003 (in Japanese). 